\title{
Performance Enhancement of Improved K-best Decoder using Gold Code for MIMO System
}

\author{
V.Tamizhamuthu \\ M.Tech II yr, \\ Department of Electronics and Communication, \\ Sri Manakula Vinayagar Engineering College, \\ Puducherry, India-605107.
}

\author{
R.Ramya \\ Assistant Professor, \\ Department of Electronics and Communication, \\ Sri Manakula Vinayagar Engineering College, \\ Puducherry, India-605107.
}

\begin{abstract}
A multiple-input-multiple-output (MIMO) system can be implemented to enhance the capacity of a wireless link. The optimal decoder is based on the maximum likelihood principle. But as the number of the antennas in the system and the data rates increase, the maximum likelihood decoder becomes too complex to use. Examples of less complex decoding techniques are sphere decoder and K-best decoders have been implemented at the price of reduced performance at the receiver. In this work, a new type of decoding algorithm called improved K-best decoding algorithm is combined with the gold code which approaches near-maximum-likelihood performance for multipleinput-multiple-output detection. In computer simulations, it will be shown that the decoder with the improved algorithm has the performance nearer to ML decoder without increase in the decoding complexity.
\end{abstract}

\section{Keywords}

MIMO, Maximum likelihood, K-best decoder, Gold code

\section{INTRODUCTION}

Wireless communications has been one of the fastest growing fields in the past few decades. This growth has primarily been because of the exponential increase in usage of mobile phones and wireless local area networks (WLAN). Future generation of wireless networks (4G) will focus on convergence of mobile phone technology, computing, internet access, and many other multimedia applications. Hence, better spectral efficiency is a pressing requirement in order to meet the demand for new applications and services. The increasing demand on voice and multimedia communications mandates future generation wireless networks to support transmission rates of several Mbps and to accommodate a very large number of users. A future wireless terminal, by guaranteeing high-speed data, will be able to connect to different networks in order to support various services such as switched traffic, IP data packets and broadband streaming services such as video. Such networks will facilitate seamless switching access between existing and future standards [1].

The primary goal of next generation wireless systems (4G) will not only be the introduction of new technologies to cover the need for higher data rates and new services, but also the integration of existing technologies in a common platform. Hence, the selection of a generic air interface for future generation wireless systems will be of great importance. Thus the new air-interface should possess a generic architecture, enabling the integration of various technologies; should possess high spectral efficiency in order to accommodate higher data rates in a given scarce spectrum, high scalability; high adaptability and reconfigurability, all at affordable costs[2].

\section{SPACE-TIME BLOCK CODES}

The STBCs are realized with the goal of finding a tradeoff between diversity and multiplexing. The data transmission rate can be increased by sending same symbols at different time instants [3]. This would save the redundancy of sending the same symbols, which in turn would increase the data rate. But, in a multipath environment, there is a possibility that the channel cannot support the data rate decided by the Space Time Block Code. The multipath fading characteristics most often results in lossy transmission, which would lead to incorrect decoding at the receiver. Apart from the most popular Alamouti code which has a code rate of 1 , there are several other codes, which maintain the orthogonal structure of the code, but with lower code rates. The following is the half rate space-time block code for a 4-Tx system. In this case, four symbols are transmitted in eight time slots. The rate of this code is therefore $1 / 2[4]$.

$$
G_{4}=\left[\begin{array}{cccc}
x_{1} & x_{2} & x_{3} & x_{4} \\
-x_{2} & x_{1} & -x_{4} & x_{3} \\
-x_{3} & x_{4} & x_{1} & -x_{2} \\
-x_{4} & -x_{3} & x_{2} & x_{1} \\
x_{1}^{*} & x_{2}^{*} & x_{3}^{*} & x_{4}^{*} \\
-x_{2}^{*} & x_{1}^{*} & -x_{4}^{*} & x_{3}^{*} \\
-x_{3}^{*} & x_{4}^{*} & x_{1}^{*} & -x_{2}^{*} \\
-x_{4}^{*} & -x_{3}^{*} & x_{2}^{*} & x_{1}^{*}
\end{array}\right]
$$

In above matrix the column specifies the antenna number and row specifies the time slot. The code rate can be increased by reducing the time slots in transmitting the same information. This can be clearly observed in the following code which has a higher code rate.

\section{GOLD CODES}

Gold codes are used efficiently when multiple data is transmitted where the codes have bounded small crosscorrelations within a set of transmitted symbols. A set of Gold code sequences consists of $2^{n}-1$ sequences each one with a period of $2^{n}-1$. 
This gold code can be generated using the following steps.

1. Pick two maximum length sequences of the same length $2^{n}-1$ such that their absolute cross-correlation is less than or equal to $2^{(n+2) / 2}$, where $n$ is the size of the linear feedback shift register used to generate the maximum length sequence [5].

2. The set of the $2^{n}-1$ exclusive-ors of the two sequences in their various phases (i.e. translated into all relative positions) is a set of Gold codes. The highest absolute cross-correlation in this set of codes is $2^{(n+2) / 2}+1$ for even $n$ and $2^{(n+1) / 2}+1$ for odd $n$. The code is given below.

$$
\begin{aligned}
C & =\frac{1}{\sqrt{1+p^{2}}}\left[\begin{array}{ll}
S_{i}+j p . S_{i+3} & p . S_{i+1}+S_{i+2} \\
S_{i+1}-p . S_{i+2} & j p . S_{i}+S_{i+3}
\end{array}\right], \\
p & =\frac{-1+\sqrt{5}}{2}
\end{aligned}
$$

The matrix given above is the transmission format where row indicates the number of antenna and column indicates the time slots required to transmit the symbols $\left\{\mathrm{S}_{\mathrm{i}}, \mathrm{S}_{\mathrm{i}+1}, \mathrm{~S}_{\mathrm{i}+2}, \mathrm{~S}_{\mathrm{i}+3}\right\} . \mathrm{p}$ is the constant which is given by above value used to transmit 4 symbols in just 2 time slots. This achieves full diversity and full rate and the code is called golden code. It is introduced in the transmitter side which encodes the symbols efficiently and obtains the full code rate.

\section{DECODER}

Maximum likelihood decoder achieves the best performance in terms of SER among all the decoding techniques[6 7]. The ML decoding technique is certainly an optimum decoding technique but with the increase in the number of transmit and receive antennas along with a complex constellation adds to the computational complexity of the decode mechanism.

Two innovative enumerations have been presented in 1985 by Fincke and Pohst and in 1994 by Schnorr and Euchner[8]. They differ by the way sorting of the nodes is performed. In the former, the nodes are sorted according to their increasing values in a unique way independent on the search performed. The latter improves sorting by ordering the nodes according to their partial search metric. The lattice decoder using the Fincke-Pohst strategy is called SD (sphere decoder), and the lattice decoder using the Schnorr-Euchner strategy is called SE [9].

Sphere decoding is a widely used technique in communications. Given a received noisy signal, this method retrieves the source signal by exhaustively searching for an optimal solution in a hyper-sphere[10]. There are many variants of the Sphere Decoder, depending on the initial radius, and the way the search points are located in the algorithm[11].

\subsection{Improved K-Best Algorithm}

In the breadth-first algorithm, as the decoder travels down the tree the nodes to be visited increases. So, the $\mathrm{K}$-best algorithm was proposed [12]. Alternative to breadth-first algorithm, in Kbest algorithm only $\mathrm{K}$ best nodes are kept in each layer which is to be visited in the next layer. However, this algorithm has performance degradation because some nodes are sacrificed. Also it has a tradeoff between performance and complexity. If the chosen $\mathrm{K}$-value is larger, then the complexity will be high. In contrast, if it is small, then the performance is poor[13].

To improve the performance of the K-best algorithm, should observe the search paths for the desired node in the tree. That desired node may not be included in the selected K-best nodes due to its larger metric. This will happen in top layers more often and the performance gets degraded. So, we use look-ahead technique which will include the desired node into the selected K-best nodes[14].

The following equation gives the Partial ED values at two consecutive layers ' $i$ ' and ' $i+1$ ' [15].

$$
\begin{aligned}
& \operatorname{PED}\left(s_{i}\right)=P E D_{i+1}\left(s_{i+1}\right)+\left|z_{i}-\sum_{j=1}^{2 M} r_{i, j} s_{j}-r_{i, j} s_{j}\right|^{2} \\
& \operatorname{PED}\left(s_{i-1}\right)=P E D_{i}\left(s_{i}\right)+\left|z_{i-1}-\sum_{j=1+1}^{M} r_{i, j} s_{j}-r_{i, j} s_{j}-r_{i-1 . j-1} s_{j-1}\right|^{2}
\end{aligned}
$$

In both equations $s_{i}$ is used which clearly says that $\operatorname{PED}\left(s_{i}\right)$ is used to compute PED of next layer. This helps to select Kvalues in layer ' $i$ '. Likewise, we can use the information of $\operatorname{PED}\left(\mathrm{s}_{\mathrm{i}-2}\right)$ when we chose the K-values in layer ' $\mathrm{s}_{\mathrm{i}-1}$ '. This technique can be used to multiple layers which enhances the performance. For example here in equation, three layers are taken to extract K-values in layer ' $i$ '.

$\operatorname{PED}\left(s_{i-2}\right)=P E D_{i-1}\left(s_{i-1}\right)+\left|z_{i-2}-\sum_{j=1+1}^{2 M} r_{i-2, j} s_{j}-r_{i-2, j} s_{j}-r_{i-2, j-1} s_{j-1}-r_{i-2, j-2} s_{j-2}\right|^{2}$

By using this technique the signal diversity can be exploited to the selected layers. To boost up the performance further we use the new type of code called gold code which attains the full rate as encoding technique.

\section{PERFORMANCE ANALYSIS}

The performance of the combined technique is verified by simulations. The independent and identically-distributed (i.i.d.) random variables are used to generate the channel. The parameters are given in below table.

Table 1. Simulation Parameters

\begin{tabular}{|c|c|}
\hline Parameters & Attributes \\
\hline No. of samples & $3 \times 10^{5}$ \\
\hline No. of Transmitting \\
Antennas
\end{tabular}


Fig. 1 presents the simulation that verifies the BER performance of gold code with space time block code whereas 4 antennas are used. From this it is clearly known gold code has better performance when compared to STBC G4. Because of 4 antennas and 8 time slots the bit error rate is poor for G4 code whereas for gold code only 2 antennas and 2 time slots are used to send two symbols. So this achieves better bit error rate performance when compared to G4 which is depicted in below fig.1.

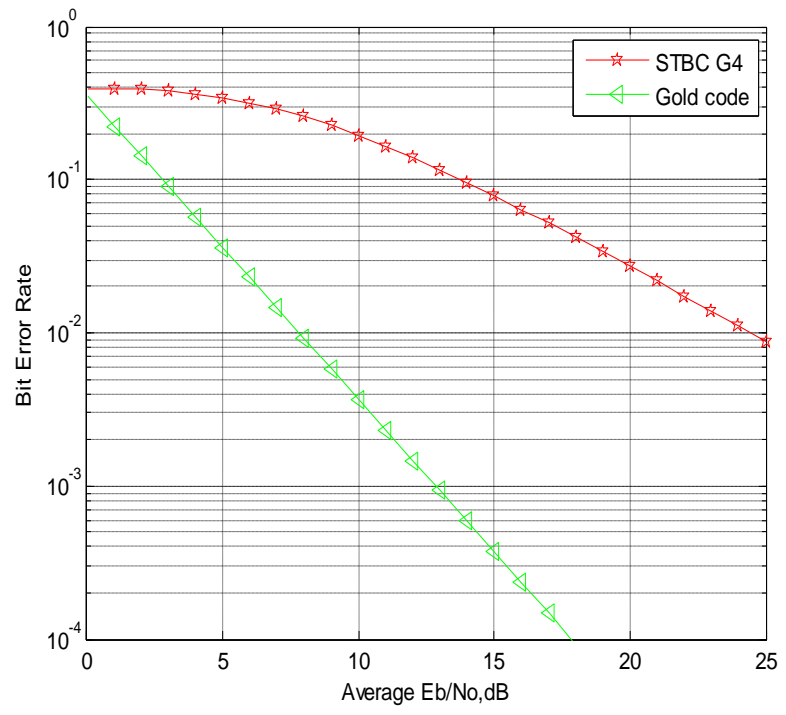

Fig. 1: BER of gold code

Fig. 2 depicts the performance of various decoders such as maximum likelihood, K-best algorithm and improved K-best with look-ahead technique. Look-ahead 1 is comparing two layers to extract $\mathrm{K}$-values while look-ahead 2 is comparing 3 layers. In those, the maximum likelihood decoder is the optimal decoder in performance, but it has high decoding complexity.

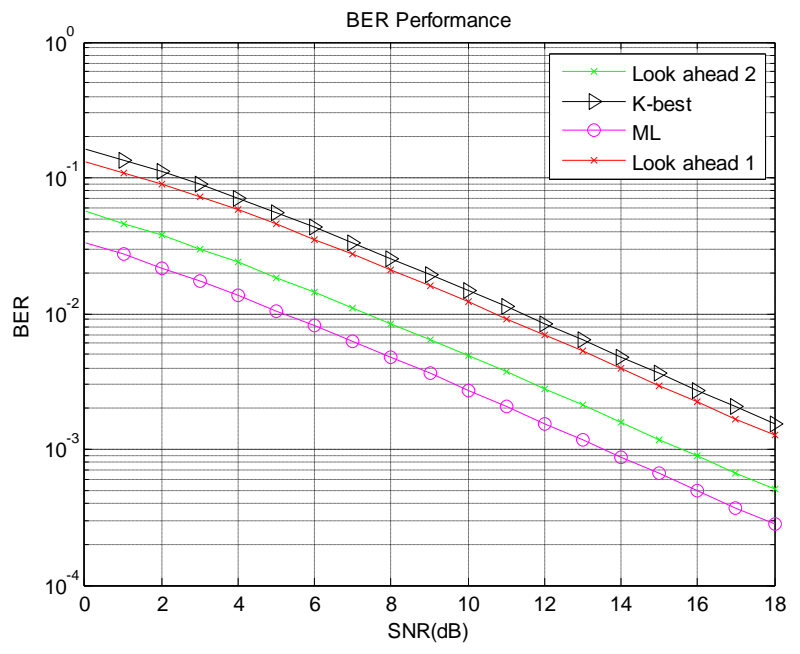

Fig 2: Performance comparison of various algorithms

The figure clearly illustrates that look-ahead technique outperforms the K-best algorithm by $4 \mathrm{~dB}$ at the BER $10^{\wedge}-2$. This means that the K-best algorithm might not be took the desired node while the look-ahead technique may take the same into account.

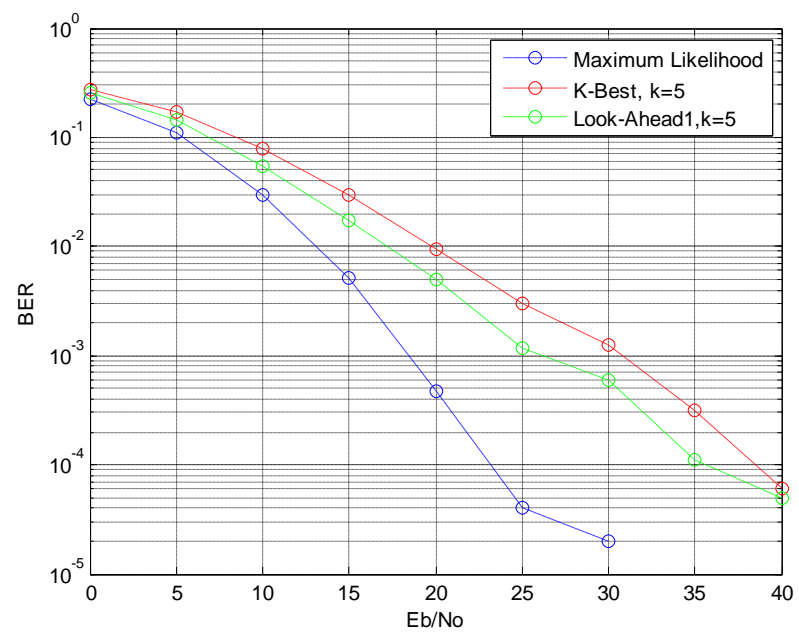

Fig 3: Performance comparison when $k=5$

In fig 3, we present the performance of K-best and look-ahead by fixing $\mathrm{K}$ as 5 . The reason to fix the value of as 5 is the tradeoff between the performance and the complexity. Also this shows the algorithm outperforms the K-best algorithm in high SNR region.

\section{CONCLUSION}

In this work, we have focused on a multiple antenna system in a flat fading environment. We compared the performance of decoders with different techniques, including maximum likelihood, K-best decoding algorithm and the improved algorithm with look-ahead technique. The performance of Kbest algorithm is enhanced by using look-ahead technique in the two and three layers combined with the gold code. In future, excellent detecting performance can be achieved by using the information of more layers to determine the survival nodes in the top layers. This consequently increases the overall performance and also decreases the complexity.

\section{REFERENCES}

[1] Helmut Blcskei and Arogyaswami J. Paulraj, "Multipleinput multiple -output (MIMO) wireless systems", pp. 90.1-90.14, 2002.

[2] Hochwald and S. Brink, "Achieving near-capacity on a multiple-antenna channel," IEEE Trans. Commun., vol. 51, no. 3, pp. 389-399Mar. 2003.

[3] H. J. Tarokh, V. and A. R. Calderbank, "Space time block codes from orthogonal designs", IEEE Transactions on Information Theory, vol. 45, no. 5, 1999.

[4] X.-B. Liang, "A High-Rate Orthogonal Space-Time Block Code", IEEE Communications Letters, vol. 7, pp. 222-223, May 2003.

[5] Ouertani, R.; Saadani, A.; Othman, G.R.-B.; Belfiore, J.C.; "On the Golden Code Performance for MIMO-HSDPA System", Vehicular Technology Conference- IEEE 64th, Sept. 2006, pp. 1-5. 
[6] E. Argell, E. Eriksson, A. Vardy, and K. Zeger, "Closest point search in lattices," IEEE Trans. Inf. Theory, vol. 48, no. 8, pp. 2201-2214, Aug. 2002.

[7] M. O. Damen, H. E. Gamal, and N. C. Beaulieu, "On maximum-likelihood detection and the search for the closest lattice point," IEEE Trans. Inf. Theory, vol. 49, no. 10, pp. 2372-2388, Oct. 2003.

[8] Z. Guo and P. Nilsson, "A VLSI architecture of the Schnorr-Euchner decoder for MIMO systems," in Proc. IEEE 6th CAS Symp. Emerging Technol.: Frontiers of Mobile and Wireless Communication, Shanghai, China, Jun. 2004, pp. 65-68.

[9] A. Burg, M. Borgmann, M. Wenk, M. Zellweger, W. Fichtner, and H. Bolcskei, "VLSI implementation of MIMO detection using the sphere decoding algorithm," IEEE Journal of Solid-State Circuits, vol. 40, July 2005, pp. 1566-1577.

[10] B. Hassibi and H. Vikalo, "On the expected complexity of sphere decoding," in Proc. Asilomar Conf. Signals, Syst., Comput, , 2001, pp. 1051-1055.
[11] A. M. Chan and I. Lee, "A New Reduced Complexity Sphere Decoder for Multiple Antenna Systems," IEEE International Conference on Communications, vol. 1, pp. 460-464,2002.

[12] Chung-An Shen, Eltawil A.M, Mondal .S and Salama .K.N, "A best-first tree-searching approach for ML decoding in MIMO system", in Proc. IEEE Int. Conf. Commun., May 30 2010,pp. 3533 - 3536.

[13] A. D. Murugan, H. El Gamal, M. O. Damen, and G. Caire, "A unified framework for tree search decoding: Rediscovering the sequential decoder," IEEE Trans. Inf. Theory, vol. 52, no. 3, pp. 933-953, Mar2006.

[14] Zhan Guo and Peter Nilsson, "Algorithm and Implementation of the K-Best Sphere Decoding for MIMO Detection", in IEEE Commun., Journal, March 2006, pp., 491-501.

[15] Pei-Yun Tsai and Xing-Cheng Lin, "Improved K-best Sphere Decoder with a Look-ahead Technique for Multiple-Input Multiple-Output Systems", in Proc. IEEE Int. Conf. Commun., May 2008,pp. 978 - 982. 